\title{
Shape based circularity measures of planar point
}

\section{sets}

\author{
Milos Stojmenović, Amiya Nayak \\ SITE, University of Ottawa, \\ Ottawa, Ontario, Canada K1N 6N5 \\ \{mstoj075, anayak\}@site.uottawa.ca
}

\begin{abstract}
We are interested in circularity measures which are invariant to rotation, scaling, and translation, are calculated very quickly and are resistant to protrusions in the data set. We propose several measures here, all of which are based on existing linearity measures that have been adapted to measure circularity. In order to make use of these linearity measures, we transfer the Cartesian coordinates of the input set into polar coordinates. The linearity of the polar coordinate set corresponds to the circularity of the original input set given a suitable center. We separately consider the circularity of ordered and unordered point sets. The circularity of unordered data is determined directly from the linearity measure, whereas the circularity of ordered data is derived by multiplying the unordered data circularity measure by a monotonicity factor. We discuss two ways of determining the center of the shape. The circularity measures are tested on a set of 25 curves. The proposed algorithms work on both open and closed curves, whereas all competing algorithms (except one) are linked with exclusively closed curves. The measures were compared with human measurements of circularity of the same set. The new methods are have been found to best correspond to human perceptions.
\end{abstract}

\section{INTRODUCTION}

We are interested in measuring how circular a finite set of points is. In analyzing various algorithms, we restrict ourselves to the following criteria. Circularity values are assigned to sets of points and these values shall be numbers in the range $[0,1]$. The circularity measure equals 1 if and only if the shape is a circle. A shape's circularity value should be invariant under similarity transformations of the shape, such as scaling, rotation and translation. The algorithms should also be resistant to protrusions in the data set. Circularity values should also be computed by a simple and fast algorithm. Circularity measures were discussed in [LS, DD, CKT, KA, P]. All of them are area based and linked to closed curves except for one: $[\mathrm{P}]$. This one is shape based and can be applied to open curves.

Here, we will propose and analyze shape based algorithms that assign circularity values to both open and closed curves, and to both ordered and unordered sets of points. These measures are adaptations of the linearity measures proposed in [SNZ]. These linearity measures are applicable since the input set of points to our circularity algorithms was transformed from Cartesian representation to polar representation, where highly circular input point sets become highly linear in the new representation, for a proper choice of center. We first consider measures for unordered point sets. The circularity of ordered sets is obtained by multiplying the unordered results by a monotonicity factor. The choice of center of each shape influences its overall circularity value. The center of each shape is traditionally seen as its center of gravity. We also consider another definition of shape center here.

One of the main advantages of our algorithms is that they work on both open and closed curves because they are shape based rather than area based. Our algorithms were tested on a set of 25 shapes. The results are compared against existing measures, and human measurements.

\section{LITERATURE REVIEW}

The most used circularity measure is the well-known $C=4 \pi A / P^{2}$, where $A$ is the area of the shape while $P$ is its perimeter. It is scale independent. However, the border of an object may be highly irregular and the calculated value of $C$ will then be very small and will not reflect the correct circularity measure, as observed in [DD].

Di Ruberto and Dempster [DD] defined new circularity measures which are translation and scale invariant and are based on mathematical morphology. They [DD] make use of a distance function $\operatorname{dist}(p)$ associated with each pixel $p$, as the integer valued radius of the smallest circle which erodes $p$. The regional maxima of the distance function represents inner points of $f$ located at the longest distance from the border of $f$. Let $h=\max \{\operatorname{dist}(p)\}$ be the maximum distance function, then the $V$ measure [DD] is defined as $V=$ sum $\operatorname{dist}(p) / h^{3}$, which is the ratio of the volume of the generated shape of the distance function and the cube of the height of the same function. $T$ measure is defined as $T=A / h^{2}$ [DD], where $A$ is the area of the object. The $E$ measure in [DD] is $E=h / \operatorname{sqrt}(A)$. The $V, T$ and $E$ measures are sensitive to intrusions, since the intrusion affects $h$, the radius of the largest circle inside the shape. In the $M$ measure in [DD], the distances from the center of gravity from the border in several directions (e.g. 8 or 16 cardinal directions) are calculated and the object deformity is calculated by the variance of these distances. This measure is not invariant to rotations and can produce a measure of 1 for non-circular objects.

Proffitt $[\mathrm{P}]$ introduced measures for circularity and ellipticity on a digital grid. His circularity measure was based on taking the mean radius $\mu_{r}$ presumably from the center of gravity to each border pixel. The standard deviation $\sigma_{r}$ of all such radii was also calculated. The proposed circularity measure was $P=\sqrt{1-\left(\sigma_{r} / \mu_{r}\right)^{2}}$. This is the only shape based measure that was found in literature.

Lee and Sallee [LS] introduce a set of area based measures of circularity, triangularity, and rectangularity. Their circularity measure first separately calculates the intersection and union of the shape area $S$ with the area of the circle $C$ that best fits the shape. Their final circularity 
measure is the ratio of the areas of the intersection and union of $S$ and $C,(S \cap C) /(S \cup C)$.

Kim and Anderson [KA] defined a compactness measure (which is a different name for a circularity measure) for convex sets as the ratio of the area of a convex region and the area of the smallest circle containing the region. The measure is then modified and applied to measuring the compactness of images with respect to digital disks. Both convex sets and digital disks are outside the scope of this research. They [KA] also observed that the perimeter calculation for digital arcs, where neighboring edges have lengths 1 or $\sqrt{2}$, measure octagonality rather than circularity in images. A similar observation was made by Bottema [Bo] who then proposed a new digital circularity measure as the ratio $1-\mid A-$ $D|/| A \mid$, where $A$ is any discrete set and $D$ is the discrete disk having the same area as $A$ and the same center of mass. $A-D$ is the set difference of $A$ and $D,(A \wedge \neg D)$, and $|X|$ is the number of pixels in $X$.

Chatzis, Kaburlasos, and Theodorides [CKT] described an image processing method to automate the particle size and shape measurement procedure. The method is based on fuzzy mathematical morphology. It is applied to estimate the size and the shape of fertilizers particles produced in the fertilizer industry. The particles tend to be spherical as much as possible. Image thresholding (the threshold is the minimal value of the smoothed histogram of the grayscale image) is applied to separate fertilized pixels from the background. Connected components were then found, and the circularity of each component is estimated as follows. The area is the number of pixels that belong to the object. The perimeter of each object is computed using a method based on fuzzy mathematical erosion. Each object is eroded two times using a $3 \times 3$ square structuring element first, and then a $3 \times 3$ crossshaped structuring element. The perimeter is then estimated using the number of eroded pixels. The equal-area diameter of an object is defined as the diameter of a circle that has area equal to the area of the object. The circularity of an object is computed as the fraction between the equal-area diameter and another "equivalent diameter" computed as the diameter of an equal perimeter circle. The circle perimeter is calculated via the erosion based method. This circularity measure tends to the unit measure when the object tends to be a perfect circle. Note that this measure is in fact, similar to the square root of the traditional circularity measure. The difference is only that the perimeter is calculated differently.

The measures that satisfy our conditions of being resistant to protrusions, while at the same time being invariant to scaling, translation and rotation are: $C, P, L S$ and CKT. $P$ is the only one that is shape based. Note that CKT in fact measures digital circularity, but is added here to approximate the circularity of ordered point sets when the points in the set are pixels with integer coordinates.

Stojmenovic, Nayak and Zunic [SNZ] proposed 6 linearity measurements for finite, planar point sets. Their measures are quickly calculated and are invariant to scale, translation or rotation. All of their methods give linearity estimates in the range $[0,1]$ after some normalization. We describe here only two out of six measures. Their Average Orientations measure takes $k$ random pairs of points along the curve. It finds their slopes $(m)$, and finds the normals to their slopes $(-m, 1)$. These normals are averaged out, and the resulting normal $(A, B)$ is deemed to be the normal to the orientation of the curve. The averaging is done separately for each vector coordinate. The measure of linearity is defined as $\sqrt{A^{2}+B^{2}}$. The contour smoothness measure was formed by taking triplets of points, and averaging out their triangular areas. Each triplet of points produced a smoothness value in the form of area/perimeter ${ }^{2}$. The maximum value for area divided by the triangle perimeter is $\sqrt{3} / 36$ (for an equilateral triangle). After smoothness values are averaged to produce value sums, the result is adjusted as follows: sums $=36$ sums $/ \sqrt{3}$. The compliment of the obtained sums value was taken as a linearity value.

\section{MEASURING CIRCULARITY}

We will present our circularity measures for both ordered and unordered point sets here. The choice of center for each shape is an important factor in measuring circularity. We have used two methods for finding the center of each shape. The first method is the general center of gravity of the shape, which corresponds to a per-coordinate average value of each pixel in the shape. The true center finding method takes the median point value of $k$ triplets sample points belonging to the curve. The results of all tests cases are presented in the next section.

The linearity measures presented in [SNZ] were used to measure circularity here. We did not modify the measures themselves. Only the input to each measure was modified. The intended input for the linearity functions was an array of $n$ Cartesian pixel coordinates in the form $\left(x_{i}, y_{i}\right)$. The Cartesian pixel array was transformed into an array of polar coordinate pixels. Points are transferred from Cartesian coordinates to Polar coordinates as follows: Point $(x, y)$ in Cartesian form would be represented by $\left(\sqrt{x^{2}+y^{2}}, \arctan (y / x)\right)$, in polar form. This form of point representation is beneficial since circular objects whose points are transferred to polar coordinates appear linear when these points are mapped. Figure 1 shows a circle with radius $r$ drawn in planar Cartesian coordinates, and its corresponding polar representation on the right.

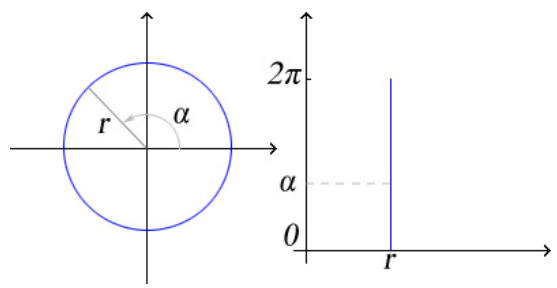

Figure 1. Cartesian and polar representations

The linearity measures of [SNZ] were used to measure the circularity of polar coordinate input sets. The algorithm that was used to test circularity of a planar set of points with a general linearity method $X$ is presented below.

Input: $\quad$ array of points: Points $=\left(X_{i}, Y_{i}\right), 1 \leq i \leq n$;

Find center of gravity $\left(X_{c}, Y_{c}\right)$ of set of points;

Translate the set by $\left(-X_{c},-Y_{c}\right)$ so that it is at the origin;

Transform set to Polar Coordinates.

$X=$ linearity value of the polar coordinate set;

$M=$ Monotonicity of point set in polar coordinates; $X M=X^{*} M$

Output: $\quad$ Circularity $X$, Ordered Circularity $M X$;

Monotonicity measures the behaviour of curves with respect to their orientation line. It is expected that monotonic curves define a more linear ordered set of points than non- 
monotonic curves. Monotonicity is introduced to 'correct' the error made by using linearity measures for unordered sets. The algorithm works by taking $N-4$ pairs of points which are 4 positions apart. A vector $v$ is found for each pair of points. Each $v$ is multiplied by the orientation line vector of the whole set of points via a dot product. The orientation line is defined via central moments [C]. If the dot product is positive, the sign $s$ of the magnitude mag of $v$ is positive, otherwise it is negative. The sum of all $s^{*} \mathrm{mag}$ is divided by the sum of the absolute values of all mag to form a monotonicity value. Monotonicity is multiplied by each linearity measure to make combined metrics that measure the linearity of ordered point sets.

The trivial way of choosing a shape's center is to take the per-coordinate average of all pixels, which is referred to as the center of gravity. This is the method that is usually chosen when measuring any shape property such as linearity or elongation. Choosing the appropriate center of a shape when measuring circularity is more delicate and heavily influences the result of the circularity measure. To illustrate this point, we turn to semi circular shape 20 . Transferring the shape to polar coordinates with respect to the center of gravity would not yield a straight line, but rather a curved one. This might result in a lower circularity measure than expected for the given shape. In order to find the 'true center' of a shape, as opposed to its traditional center of gravity, we sampled $k$ triplets of points from its point set. From each triplet, we found the center $\left(X_{t c}, Y_{t c}\right)$ that the points define. It is expected that each triplet of points will yield a different center $\left(X_{i}, Y_{i}\right)$. To choose a unique center for the shape, we individually sort the $k$ center values per coordinate, and choose the median per coordinate value to be the true center, $\left(X_{t c}, Y_{t c}\right)$. We have experimented with both types of center finding methods in this work.

\section{EXPERIMENTAL DATA}

The circularity algorithms were tested on a set of 25 non trivial shapes. These shapes were assembled by hand and are meant to cover a wide variety of non trivial cases. Each shape comprises between 300 and 700 pixels. All shapes are digital, which is why the circularity measures are not ideal.

Four sets of measurements are presented here. One set is for the circularity measure where the center of the shape is chosen to be its center of gravity and the points are unordered, the next is the case where the center of gravity is chosen to be the 'true center' and the points are unordered, the third and fourth measures are identical to the first two except that the points of each shape are ordered. In order to get an idea of which measure best measures circularity, we compared them to human measurements of circularity. A group of 20 colleagues volunteered to give their analysis of the shapes in Figure 2. Each measure was correlated to the average human results to find the one that best models them. Figure 2 shows the test curves used in the circularity experiments.

Table I holds the circularity values as measured by each method on unordered point sets. The actual measures are in the interval $[0,1]$, but they are presented as integers in the interval $[0,100]$ in the tables to save space. The $A O C$ column refers to the Average Orientations Circularity measure that takes the center of gravity as the center of each shape. $A O T$ is the average orientations circularity measure that adopts the 'true center' method when calculating circularity. The CSC column refers to the Contour Smoothness Circularity measure that takes the center of gravity as the center of each shape. CST is the Contour Smoothness circularity measure that adopts the 'true center' method when calculating circularity. The $P$ column shows the results of the measure proposed by $[\mathrm{P}]$, and the 'center of gravity' was used as the shape center. The CKT column shows the results of the measure proposed by [CKT]. $L S$ is the circularity measure proposed by [LS]. The $C$ column shows the results of the standard measure of circularity. The perimeter of each shape was calculated as in [KA]. The HM column depicts the average human perception of the circularity of each shape. The correlation values of each measure with human perception are found at the bottom of the table.

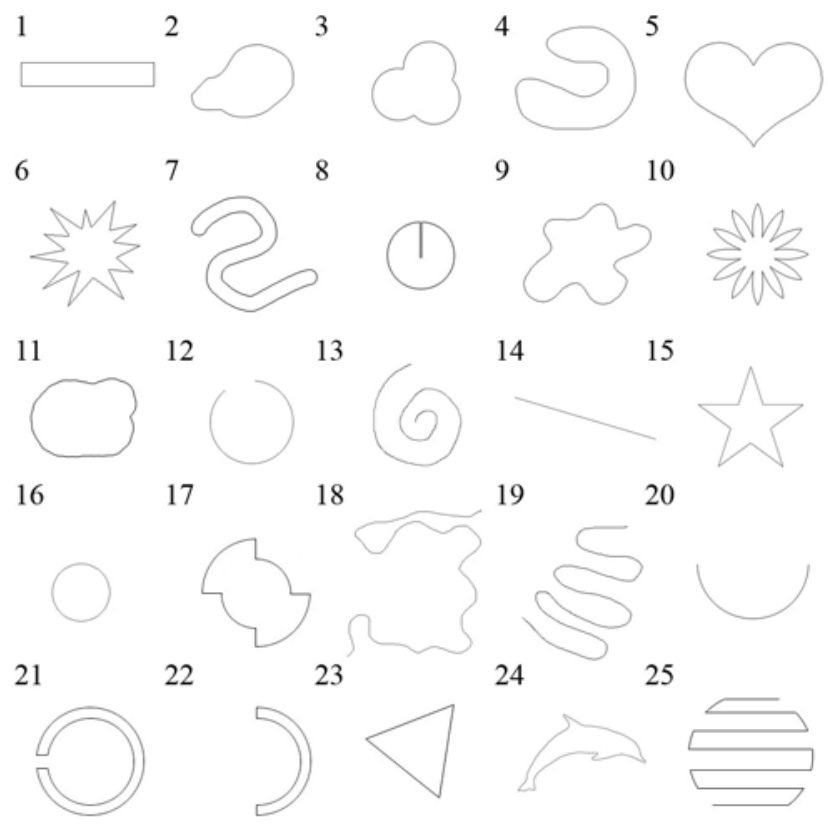

Figure 2. Circularity test set

TABLE I. CIRCULARITY RESULTS FOR UNORDERED POINT SETS

\begin{tabular}{r|cc|cc|cccc|c|}
\multicolumn{1}{c}{} & AOC & AOT & CSC & CST & P & CKT & LS & C & HM \\
\cline { 2 - 9 } 1 & 51 & 50 & 32 & 32 & 88 & 69 & 30 & 42 & 12 \\
2 & 86 & 83 & 61 & 59 & 98 & 91 & 72 & 78 & 77 \\
3 & 90 & 89 & 69 & 67 & 99 & 90 & 79 & 76 & 64 \\
4 & 74 & 70 & 38 & 36 & 94 & 62 & 53 & 36 & 61 \\
5 & 75 & 74 & 46 & 46 & 98 & 85 & 70 & 68 & 45 \\
6 & 77 & 71 & 51 & 48 & 98 & 36 & 67 & 13 & 40 \\
7 & 74 & 75 & 50 & 50 & 93 & 39 & 18 & 15 & 15 \\
8 & 81 & 80 & 69 & 70 & 92 & 77 & 95 & 55 & 97 \\
9 & 75 & 74 & 51 & 49 & 98 & 72 & 69 & 50 & 44 \\
10 & 76 & 77 & 48 & 48 & 97 & 29 & 64 & 10 & 53 \\
11 & 96 & 96 & 81 & 82 & 99 & 96 & 80 & 85 & 85 \\
12 & 99 & 99 & 90 & 98 & 100 & & & & 94 \\
13 & 68 & 66 & 39 & 36 & 93 & & & & 65 \\
14 & 9 & 88 & 30 & 83 & 82 & & & & 0 \\
15 & 66 & 66 & 34 & 32 & 96 & 53 & 61 & 26 & 27 \\
16 & 100 & 100 & 98 & 99 & 100 & 99 & 98 & 93 & 100 \\
17 & 78 & 89 & 72 & 71 & 98 & 82 & 66 & 60 & 38 \\
18 & 59 & 66 & 35 & 40 & 98 & & & & 12 \\
19 & 60 & 57 & 36 & 29 & 93 & & & & 4 \\
20 & 75 & 99 & 61 & 95 & 93 & & & & 62
\end{tabular}




\begin{tabular}{c|cc|cc|cccc|c|}
21 & 91 & 89 & 76 & 76 & 99 & 33 & 0 & 12 & 91 \\
22 & 76 & 86 & 64 & 77 & 94 & 44 & 12 & 21 & 56 \\
23 & 86 & 89 & 70 & 71 & 97 & 79 & 69 & 59 & 20 \\
24 & 45 & 44 & 31 & 20 & 90 & 52 & 41 & 25 & 29 \\
25 & 75 & 73 & 53 & 48 & 94 & & & & 43 \\
\hline cor & 0.74 & 0.58 & 0.75 & 0.56 & 0.52 & 0.33 & 0.35 & 0.42
\end{tabular}

We see that some of the entries in the $C K T, L S$ and $C$ columns are empty because these measures are not defined for open shapes. Even when omitting these open curves from consideration, we see that the first four measures more closely follow the average human measurements.

Table II shows the results of the circularity measures as applied to ordered data. The $A O M$ column represents the average orientations circularity measure multiplied by its corresponding monotonicity factor. AOTM shows the average orientation circularity considering the 'true center' of each shape, multiplied by the corresponding monotonicity. The CSM column represents the Contour Smoothness circularity measure multiplied by its corresponding monotonicity factor. CSTM shows the Contour Smoothness circularity considering the 'true center' of each shape, multiplied by the corresponding monotonicity. Column $P M$ shows the $P$ circularity measure multiplied by the monotonicity values of each shape. This is the only other measure, apart from our own, that monotonicity was applicable to since other existing measures area based, where the order of the pixels is not defined. The other measures are area based, and monotonicity cannot be calculated for them. The monotonicity values, with respect to the center of gravity of each shape, are seen in column $M O N$. The $H M M$ column shows the average human measurements of circularity considering the monotonicity of shapes.

TABLE II. CIRCULARITY RESULTS FOR ORDERED POINT SETS

\begin{tabular}{r|cc|cc|c|c|c|}
\multicolumn{1}{c}{ AOM } & AOTM & CSM & \multicolumn{1}{c}{ CSTM } & PM & MON & HMM \\
\cline { 2 - 7 } 1 & 51 & 42 & 32 & 27 & 88 & 1.00 & 8 \\
2 & 86 & 83 & 61 & 59 & 98 & 1.00 & 75 \\
3 & 90 & 89 & 69 & 67 & 99 & 1.00 & 63 \\
4 & 23 & 23 & 12 & 12 & 30 & 0.32 & 20 \\
5 & 75 & 74 & 46 & 46 & 98 & 1.00 & 38 \\
6 & 74 & 68 & 49 & 46 & 93 & 0.95 & 30 \\
7 & 30 & 30 & 20 & 20 & 38 & 0.41 & 8 \\
8 & 28 & 28 & 24 & 25 & 32 & 0.35 & 80 \\
9 & 75 & 74 & 51 & 49 & 98 & 1.00 & 36 \\
10 & 76 & 77 & 48 & 48 & 97 & 1.00 & 37 \\
11 & 96 & 96 & 81 & 82 & 99 & 1.00 & 87 \\
12 & 35 & 25 & 32 & 24 & 60 & 0.60 & 93 \\
13 & 8 & 9 & 5 & 5 & 12 & 0.12 & 54 \\
14 & 0 & 1 & 0 & 1 & 10 & 0.13 & 1 \\
15 & 66 & 65 & 34 & 32 & 96 & 1.00 & 25 \\
16 & 100 & 100 & 98 & 99 & 100 & 1.00 & 100 \\
17 & 64 & 30 & 59 & 24 & 81 & 0.82 & 46 \\
18 & 5 & 8 & 3 & 5 & 24 & 0.25 & 10 \\
19 & 2 & 0 & 1 & 0 & 3 & 0.04 & 4 \\
20 & 28 & 0 & 22 & 0 & 93 & 1.00 & 65 \\
21 & 29 & 30 & 24 & 26 & 32 & 0.32 & 38 \\
22 & 31 & 41 & 26 & 37 & 38 & 0.41 & 26
\end{tabular}

\begin{tabular}{c|cc|cc|c|c|c|}
23 & 86 & 89 & 70 & 71 & 97 & 1.00 & 23 \\
24 & 21 & 2 & 14 & 1 & 42 & 0.47 & 16 \\
25 & 7 & 7 & 5 & 5 & 9 & 0.10 & 16 \\
\cline { 2 - 8 } cor & 0.49 & 0.43 & 0.61 & 0.55 & 0.44
\end{tabular}

Considering both tables seen above, we can say that the circularity of unordered sets is much more easily agreed upon by both humans and computers. One of the most unexpected results that occurs in our measurements is the circularity value of shape 14 when measured by the AOT and CST methods. The resultant circularity is extremely high (0.88) for a straight line segment. The reason this happens is that the 'true center' for such a line segment is found to be vary far away from the point set. This line is highly non-circular, as confirmed by the human measurements, yet the true center assigned to it makes it seem part of a large circle, and hence drastically upgrades its circularity value. In order to avoid such discrepancies, the shape must cover a larger percentage of the overall circle to be considered circular.

Calculating all of the new measures was done very quickly on a $2.4 \mathrm{GHz}$ AMD Athlon platform. The results of both tables are generated in less than 1 second by the computer.

Overall, the measure that best correlated with human perception on unordered point sets was SCS. For ordered sets, CSM proved most compatible. Aside from our own proposed method, the $P$ measure was the closest competitor from the literature.

\section{ConClusion}

Recently, there have been several applications that concentrate on finding circular objects in images. They range from identifying onions in gardens, to finding tires on cars when seen from the side. Future applications might include identifying individual cells based on their boundaries in medical imaging.

\section{REFERENCES}

[Bo] M.J. Bottema, Circularity of objects in images, Proc. IEEE Acoustic, speech and signal processing, ICASSP, Istanbul, 2000.

[C] D. Csetverikov, Basic algorithms for digital image analysis, Course, Institute of Informatics, Eotvos Lorand University, visual.ipan.sztaki.hu.

[CKT] V. Chatzis, V.G. Kaburlasos, M. Theodorides, An image processing method for particle size and shape estimation, Proc. 2rd International Scientific Conference on Computer Science, Chalkidiki, Greece, 30 September - 2 October 2005.

[DD] C. Di Ruberto and A. Dempster, Circularity measures based on mathematical Morphology, Electronics Letters, Vol. 38, No. 20, 1691-1693, September 2000.

[KA] C. E. Kim, T. A. Anderson: Digital Disks and a Digital Compactness Measure, Proc. of the sixteenth annual ACM symposium on Theory of STOC 1984: 117-124.

[LS]D.R. Lee, T. Sallee, A method of measuring shape, Geographical Review, Vol. 60, No. 4, pp. 555-563, 1970.

[P] D. Proffitt, The measurement of circularity and ellipticity on a digital grid, Pattern Recognition, .15, 5, pp. 383-387, 1982.

[SNZ] M. Stojmenovic, A. Nayak, J. Zunic, Measuring Linearity of a Finite Set of Points, IEEE Int. Conf.. Cybernetics and Intelligent Systems (CIS) and Robotics, Automation and Mechatronics (RAM), pp. 222-227, Bangkok, Thailand, 7-9 June 2006. 